\title{
Scaled Momentum Spectra in the Current Region of the Breit Frame at HERA
}

\author{
Beata Brzozowska \\ On behalf of the ZEUS Collaboration \\ University of Warsaw - Institute of Experimental Physics \\ Hoża 69, 00-681 Warsaw - Poland
}

\begin{abstract}
Charged particle production has been studied in deep inelastic scattering using an integrated luminosity of $0.5 \mathrm{fb}^{-1}$ taken with the ZEUS detector at HERA. Scaled momentum distributions in intervals of $Q^{2}$ were investigated in the current region of the Breit frame. The fragmentation properties of the struck quark in DIS are compared with recent next-to-leading order calculations with the fragmentation functions obtained from $e^{+} e^{-}$experiments. Scaling violations are observed.
\end{abstract}

\section{Introduction}

In this paper parton fragmentation and hadronisation are studied using the inclusive charged particle spectrum in the current region of the Breit frame in deep inelastic scattering (DIS). The Breit frame [2] gives the best separation of the scattered quark fragments from the proton remnant.

The exchanged virtual boson is completely space-like and has longitudinal momentum $-Q$. The exchanged virtual photon collides elastically and head on with a quark carrying a longitudinal momentum of $Q / 2$. The struck quark is scattered with equal but opposite momentum. The particles produced in the Breit frame can be assigned to one of two regions: the current region if their longitudinal momenta in the Breit frame are negative, and the target region if their longitudinal momenta are positive. The maximum momentum of a particle in the current region of this frame is $Q / 2$.

In the Breit frame the scaled momentum variable $x_{p}$ is defined to be equal $2 p_{h} / Q$, where $p_{h}$ is the momentum of a hadron measured in the Breit frame. This variable is transformed to $\xi_{p}=\ln \left(1 / x_{p}\right)$ when comparing the data with theoretical calculations.

Recently accumulated high statistics of $e p$ interaction permits the scaled momentum distributions to be studied as a function of $Q$ and $x$ in the range $0.002<x<0.75$ and $160<Q^{2}<40960 \mathrm{GeV}^{2}$. Together with the previous results [3] at $10<Q^{2}<160 \mathrm{GeV}^{2}$ the evolution of fragmentation functions is shown within a single experiment over a wide range of $Q$.

The data presented here were collected during the 1996-2007 running period at the electron-proton collider HERA using the ZEUS detector. The data of this analysis corresponded to an integrated luminosity of $0.5 \mathrm{fb}^{-1}$. A detailed description of the ZEUS detector can be found elsewhere [4].

The reconstructed tracks in the laboratory frame used in this analysis were associated with the primary event vertex and had $p_{T}>150 \mathrm{MeV}$ and $|\eta|<1.75$, where $\eta$ is the pseudorapidity given by $-\ln (\tan (\theta / 2))$ with $\theta$ being the polar angle of the measured track with respect to the proton direction. This was a region of high CTD acceptance, where the detector response and systematics were best understood.

Neutral current DIS events were generated using ARIADNE 4.12 [6] and LEPTO 6.5.1 [5] programs interfaced with the HERACLES [7] program for radiation correction. ARIADNE 
uses the parton cascade modelled with the colour-dipole model (CDM) and for the hadronisation phase the Lund string-fragmentation model [8]. Another approach to model the parton cascade was included in LEPTO which incorporated the LO matrix element matched to parton showers (MEPS). This program also used the Lund string fragmentation model.

The distributions generated by Monte Carlo (MC) models included the products of strong and electromagnetic decays, but excluded the decay products of weakly decaying particles with lifetime greater than $3 \times 10^{-11} \mathrm{~s}$.

\section{Scaled momentum spectra}

The scaled momentum distributions are investigated as a function of $Q$ and $x$, in the range $0.002<x<0.75$ and $160<Q^{2}<40960 \mathrm{GeV}^{2}$. Results are presented in Figs 1-3 together with the previous ZEUS results of $10<Q^{2}<160 \mathrm{GeV}^{2}[3]$. Figures show the data points with statistical and systematic uncertainties.

The inclusive charged particle distributions, $1 / \sigma d \sigma / d \xi_{p}$ in the current region of the Breit frame, where $\sigma$ is the DIS cross section in the chosen intervals of $Q^{2}$, are shown in Fig. 1 .

The scaled momentum distributions are approximately Gaussian in shape. As $Q^{2}$ increases, the multiplicity increases and, in addition, the peak of the distribution moves to larger $\xi_{p}$ values. The suppression of hadron production at very small $x_{p}$ or large $\xi_{p}$ is a consequence of the destructive interference of soft gluons.

The MLLA predictions $[9,10]$ in Fig. 1 are calculated with two free energy-independent parameters adjusted to values deduced from the LEP data $(\Lambda=$ $Q_{0}=270 \mathrm{MeV}$ for the effective energy scale and $K_{h}=$ 1.31 for the LPHD hypothesis). The obtained limiting spectra give a reasonable description of the shape at $80<Q^{2}<1280 \mathrm{GeV}^{2}$. In the low $Q^{2}$ regions the average multiplicity is lower than predicted by MLLA which is interpreted by significant migrations

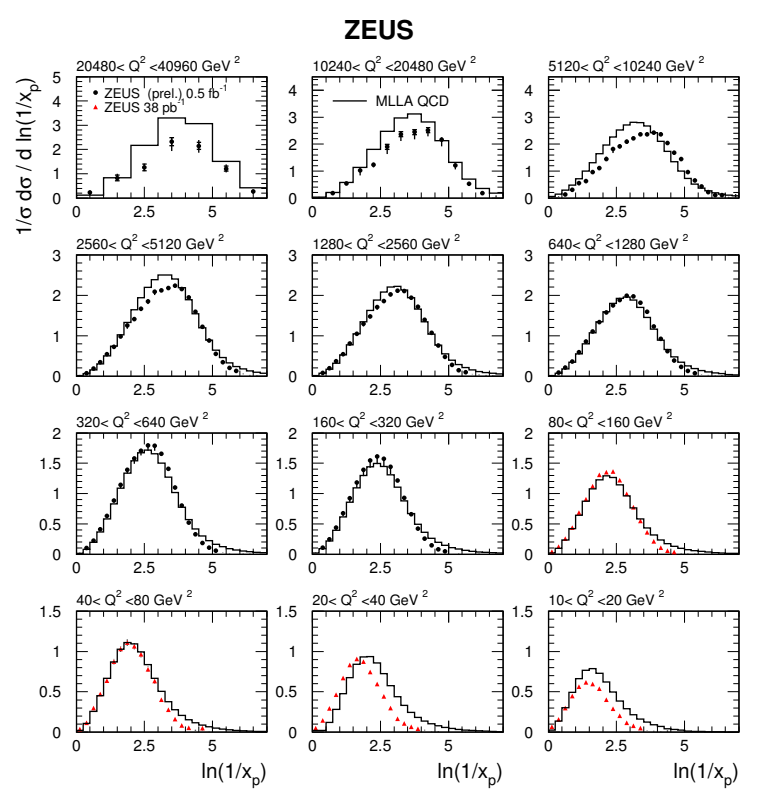

Figure 1: The charged particle distributions. The triangles points come from [3]. The full line represents the MLLA predictions. of particles to the target region of the Breit frame. As $Q^{2}$ increases the peaks are shifted more than expected towards high $\ln \left(1 / x_{p}\right)$. We conclude that the analytical MLLA QCD calculations under LPHD assumption cannot describe the data in the entire range of $x_{p}$ and $Q^{2}$.

The scaling violations can be seen more clearly if the data are plotted in bins of fixed $x_{p}$ as a function of $Q^{2}$. 
Figure 2 shows that the distributions rise with $Q^{2}$ at low $x_{p}$ and fall-off at high $x_{p}$ and high $Q^{2}$. Below $Q^{2}=80 \mathrm{GeV}^{2}$ (see Fig. 1) the fall-off is due to depopulation of the current region discussed before. These regions are relatively well reproduced by ARIADNE and LEPTO predictions. At the higher $Q^{2}$ regions and higher $x_{p}$ the data fall down faster than predicted by leading order MC models with parton shower included. The statistical precision of the data prevents any stronger statement to be made. No tuning of the Monte Carlo parameters has been performed to improve the agreement with the data.

Figure 3 shows the comparisons with the NLO calculations. Generally, the calculations do not describe the data well. The data are overlaid with predictions of the available FF parametrisations $[11,12,13]$ fitted from $e^{+} e^{-}$ data. The parametrisations are calculated for $x_{p}>0.1$, where the theoretical uncertainties are small and unaffected by the hadron mass effects which are not included in the fragmentation function. The logarithmic energy slope in the ep data is steeper than in all the parametrisations. At low $x_{p}<0.2$ all predictions fail to describe the data. We conclude that the scaling violation in the $e p$ data is stronger than predicted.

The observed discrepancy between data and parametrisations as well as the inconsistency with the MC models at high $Q^{2}$ may suggest that a contribution from heavy-flavor states may be different than in $e^{+} e^{-}$collisions.

\section{Conclusions}

Charged particle distributions have been measured in the current region of the Breit frame in DIS over a wide range of $Q^{2}$ values and show clear evidence in a single experiment for scaling violations in scaled momenta as a function of $Q^{2}$. They support the hypothesis of the coherent nature of QCD cascade. The data are neither well described by the MLLA calculations together with LPHD nor with NLO calculations. The leading order MC models with parton shower included need to be improved to reproduce the measured data at the highest and lowest $Q^{2}$ values.

Author thanks the DESY Directorate for the financial support and Dr. S. Albino for providing the theoretical calculations. 


\section{ZEUS}
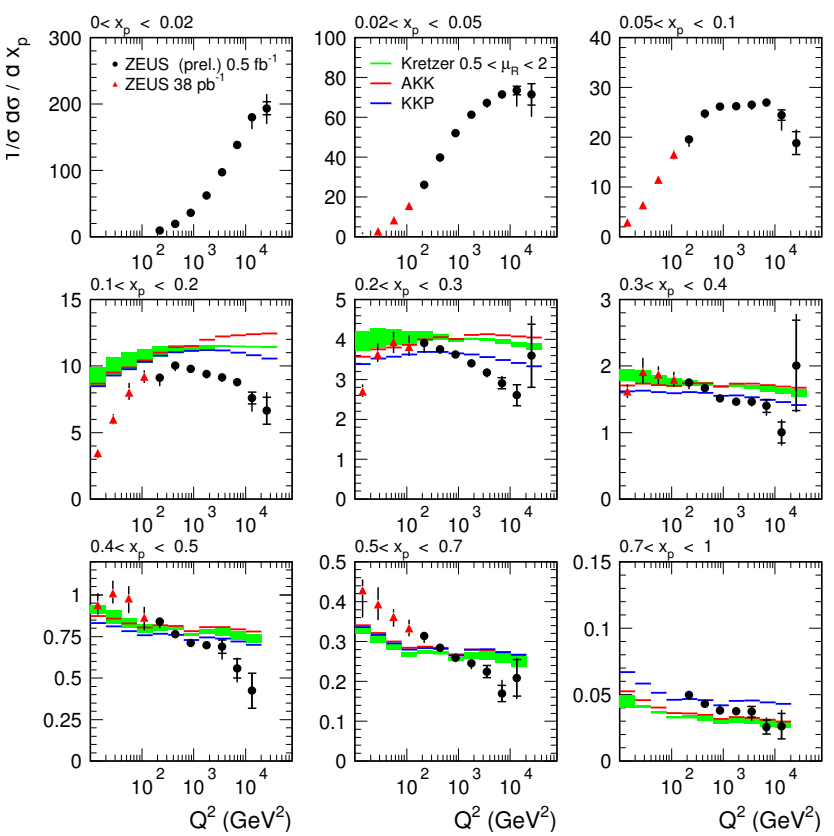

Figure 3: The inclusive charged particle distributions $1 / \sigma d \sigma / d x_{p}$ as in Fig. 2. The wide (green) lines represent Kretzer's predictions, the thin and light (blue) lines represent KniehlKramer-Pötter's predictions improved recently by Albino-Kniehl-Kramer and marked as the thin and dark (red) lines.

\section{References}

[1] Slides: http://indico. cern. ch/contributionDisplay . py? contribId=232\&sessionId=6\&conf Id=9499

[2] R.P. Feynman, Photon Hadron Interactions (1972).

[3] ZEUS Collaboration, M. Derrick et al., Z. Phys. C 6793 (1995). ZEUS Collaboration, J. Breitweg et al., Phys. Lett. B 414428 (1997). ZEUS Collaboration, J. Breitweg et al., Eur. Phys. J. C 11258 (1999).

[4] ZEUS Collaboration, U. Holm The ZEUS Detector, Status Report (unpublished), DESY (1993), available on http://www-zeus.desy.de/bluebook/bluebook.html.

[5] G. Ingelman, A. Edin and J. Rathsman, Comp. Phys. Comm. 101108 (1997).

[6] L. Lönnblad, Comp. Phys. Comm. 7115 (1992).

[7] A. Kwiatkowski, H. Spiesberger and H.J. Möhring, Comp. Phys. Comm. 69155 (1992).

[8] B. Andersson et al., Phys. Rep. 9731 (1983).

[9] V.A. Khoze, W. Ochs, Int. J. Mod. Phys. A 122949 (1997).

[10] Y.L. Dokshitzer, V.A. Khoze, A. Mueller and S. Troyan, Basics of Perturbative QCD, Editions Frontiéres, Gif-sur-Yvette, France (1991).

[11] S. Kretzer, Phys. Rev. D 62054001 (2000).

[12] B.A. Kniehl, G. Kramer, B. Pötter, Phys. Rev. Lett. 855288 (2000).

[13] S. Albino, B.A. Kniehl, G. Kramer, NUcl. Phys. B 725181 (2005). 